\title{
Mutation to second sphere residue in peptidylglycine $\alpha$-hydroxylating monooxygenase (PHM) reveals role of hydrogen-bonding network RENEE ARIAS ${ }^{1}$, Ninian Blackburn ${ }^{2}$ \\ ${ }^{1}$ Oregon Health and Science University ${ }^{2}$ Oregon Health and Science University ariasr@ohsu.edu
}

Peptidylglycine $\alpha$-amidating monooxygenases (PAMs) and their associated family of copper monooxygenases are essential for the biosynthesis and modification of many signaling peptides, particularly neuroendocrine peptides. The hydroxylase domain (PHM) catalyzes the first step in peptide amidation by hydroxylation of the glycineextended peptide substrate. PHM accomplishes hydroxylation via the crucial transfer of two electrons from one copper center $(\mathrm{CuH}$, also called the $\mathrm{H}$-site) to the oxygen-binding and catalytic center (CuM, M-site) over $11 \AA$ in one turnover event. The M-site is coordinated by H242, H244, and a weak, yet catalytically essential, interaction with M314. The H-site (coordinated by H107, H108, and H172), is hypothesized to supply to reducing electrons to the M-site via long range electron transfer (ET). An important and unresolved factor driving the efficiency of catalysis in PHM is the chemical structure and reactivity of the initial copper-oxygen intermediate. Correspondingly, the presence of a high-valent copper-oxygen intermediate requires significant enzyme stabilization. We show strong evidence that the purported intermediate is stabilized by a strong hydrogen-bonding network, mediated by the amide oxygen of side chain Q272. Our mutant, Q272E, exhibits a strong hydrogen bond (2.5 $\AA$ ) with H242, and significantly alters the biochemistry of the enzyme, as well as the overall structure.

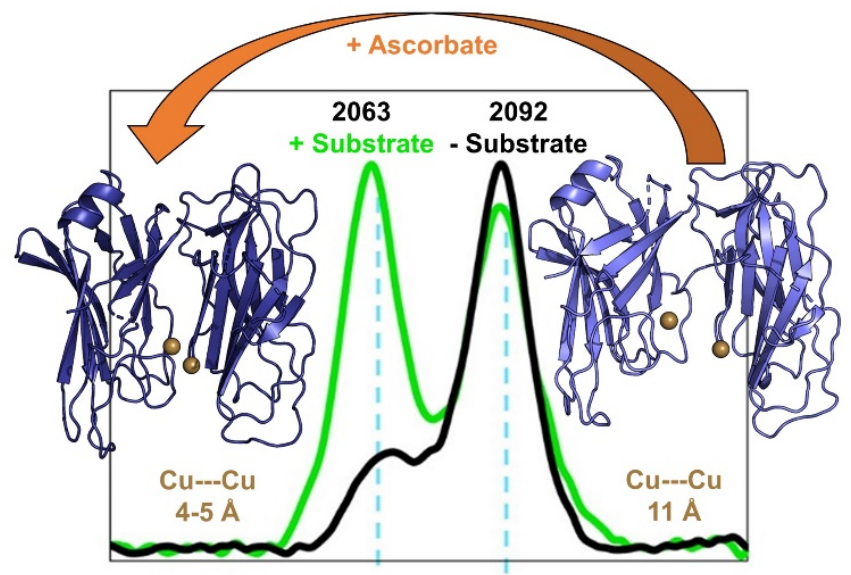

Wavenumber $\left(\mathrm{cm}^{-1}\right)$

Figure 1 